\title{
Diagnostic Value of Novel Presepsin and Inflammatory Biomarkers in Predicting the Clinical Course of COVID-19
}

\author{
๑ Faraz Hasan Mirza, ๑ Faraz Ahmed Baig, ๑ Serajuddaula Syed \\ Ziauddin University, Department of Pathology, Karachi, Pakistan
}

Abstract

Aim: The diagnostic value of inflammatory markers to determine the severity of Coronavirus disease-2019 (COVID-19) is postulated in some recent studies, but conclusions were inconsistent. Hence, we intend to examine the utility of presepsin, procalcitonin and C-reactive protein in predicting the severity of COVID-19 infection.

Methods: Single-center cross-sectional study was undertaken at the Intensive care unit of a university hospital. Eighty consecutive cases diagnosed with Severe Acute Respiratory syndrome-Coronavirus-2 RNA between October 2020 and July 2021 were classified according to the severity of the disease. Laboratory data related to Procalcitonin and C-reactive protein was retrieved from investigations coinciding with the day of admission. The stored plasma was subjected to an enzyme-linked immunosorbent assay to determine plasma presepsin levels. Statistical test for measures of screening was employed and a receiver operator curve was generated.

Results: We have determined that presepsin is the most sensitive prognostic indicator (93.3\%) with a strong statistical association $(p<0.001)$ for COVID-19. $15.99 \mathrm{ng} / \mathrm{L}$ could be used as a reference level to predict the progressive clinical course. Relatively lower sensitivity $(88 \%)$ and positive statistical correlation $(p=0.049)$ of C-reactive protein with high-risk infection were also observed. Procalcitonin showed limited diagnostic and prognostic value in our series.

Conclusion: Our findings seem to demonstrate the role of presepsin in providing prognostic information in COVID-19 patients. Therefore, we suggest that early monitoring of presepsin with routine marker profile might help in identifying patients suffering from a more severe disease.

Keywords: SARS-CoV-2 patients, biomarkers, presepsin, prognosis

\section{Introduction}

The Coronavirus disease-2019 (COVID-19) pandemic has become a major health concern across the globe resulting in at least 4.1 million deaths to date secondary to COVID-induced pneumonia and septicemia (1). According to CDC guidelines, a vast number of COVID-19 cases exhibit mild to moderate clinical course without the need for hospitalization. However, around $19 \%$ of cases suffer from severe disease reflected by dyspnea, hypoxia with up to $50 \%$ lung involvement. Unfortunately, $5 \%$ of these severe cases may progress to a critical stage, complicated by respiratory failure, shock, or multiorgan system dysfunction (2). Therefore, early diagnosis and predicting the severity of the disease is the key to implementing appropriate therapeutic interventions that would eventually improve outcomes in patient. In this regard, laboratory medicine lies at the core of diagnosing and monitoring the vast majority of human diseases including COVID-19 (3).

The tools presently available in laboratory medicine for diagnosing COVID-19 include molecular testing, serological testing and inflammatory biomarkers (4). Among these tools, molecular testing has certain limitations such as; anatomic variations in the patient, inadequate sample collection and storage, genetic changes in the virus and use of unsuitable reference range. Also, the biological source used to quantify the viral RNA, have a low detection rate and is not easy to obtain (5). Similarly, serological testing has the disadvantage of giving positive results after a month of the onset of symptoms (6). This leaves us with the choice of inflammatory biomarkers which are easy to

Address for Correspondence: Faraz Ahmed Baig, Ziauddin University, Department of Pathology, Karachi, Pakistan 
quantify, cost-effective, have better accuracy, potential to predict disease severity and are measured in a biological material that is easy to procure such as blood (7).

In this regard, inflammatory biomarkers including procalcitonin (PCT), C-reactive protein (CRP) and recently discovered presepsin (P-SEP) has shown promising results to predict disease severity in recent studies (8). Hence, we have taken a novel initiative to conduct large-scale original research with the aim to determine the diagnostic value of P-SEP, PCT and CRP in predicting the clinical course of COVID-19 infection and also sought the association of these molecules with the progressive clinical course of infection. The outcome of this study may prove vital in identifying novel molecules that could serve as an indicator to predict rapidly progressive COVID-19 infection and also identify patients presented with advanced underlying disease.

\section{Methods}

\section{Study Design and Ethical Considerations}

A single-center, cross-sectional study on 80 clinically proven COVID-19 cases was undertaken at a tertiary care set-up in Karachi, Pakistan. Ethical approval was sought from Ethics Review Committee (1701219FHPAT) in February 2020 for the collection of the cases diagnosed with COVID-19 infection. Written informed consent was obtained from all participants.

\section{Sampling}

All patients were receiving in-patient care at ICU and isolation wards of various tertiary care hospitals from October 2020 to July 2021. The diagnosis of COVID-19 was done using sensitive PCR and the subjects were selected using a non-probability consecutive sampling model. The cases were characterized into 2 groups: Moderate, and severe to critical, based on the clinical course according to CDC guidelines. Patients with the outcome of death were excluded from this study.

\section{Data Collection}

The clinical data of lab investigation including blood culture, PCT and CRP at day 0 of admission and demographic data, age and gender were obtained from the hospital record, while the stored blood drawn at day 0 of admission for routine workup was also retrieved from specimen storage facility of the lab for P-SEP analysis.

\section{Laboratory Assessments}

The frozen plasma was allowed to melt and $3 \mathrm{cc}$ was subjected to an enzyme-linked immunosorbent assay (ELISA) for P-SEP, using a commercially available kit (Bioassay technology; cat. no E3754Hu). The reaction was performed according to manufacturer protocol in a multidisciplinary BSL-2 category lab following WHO prescribed standard operating procedures (SOP) (9). Briefly, 3cc plasma was centrifugated at $3000 \times \mathrm{g}$ for 10 min. ELISA assay was done at room temperature by adding standard, streptavidin-HRP, sample, anti-lgA antibodies into the standard well. The well was then covered with sealer and the sample was incubated for 60 mins at $37^{\circ} \mathrm{C}$. Then, the sealer was removed and the plate was washed with wash buffer. The plate was then blotted onto paper towels and substrate solutions A\&B were then added to each well. Finally, for color change stop solution was added and the optical density at $450 \mathrm{~nm}$ was determined by a microplate reader.

\section{Statistical Analysis}

For statistical analysis, SPSS version 25.0 was used. The data were analyzed for normality by Kolmogorov-Smirnov test and the Shapiro-Wilk test and median was taken as the determinant of central tendency. Crosstabulation was performed for measures of screening i.e; sensitivity, specificity, positive predictive value (PPV) and negative predictive value (NPV). Receiver operator curve (ROC) was generated and the area under the curve and cutoff levels which corresponds to severe infection were estimated. The association of biomarkers with COVID-19 was assessed and $\mathrm{p}<0.05$ was considered statistically significant. 95\% confidence interval (Cl) was used for all statistical calculations.

\section{Results}

Overall, 60 participants were in a state of severe to a critical disease whereas the remaining 20 reflected a moderate pattern. Fever and dry cough were universally present in all subjects as the chief complaint. Severe to critically ill patients showed extensive lung damage on radiology and requires intubation or mechanical ventilation to deal with hypoxia while those suffering from moderateintensity required minimal oxygen support to maintain saturation.

Table 1 presents statistical estimates of all participants included in this research. Among all patients, most subjects were men and aged above 60 years. The median age was recorded as 67.5 years. The median levels for P-SEP, PCT and CRP among patients within the severe disease group were observed as; $55.05 \mathrm{ng} / \mathrm{L}, 1.25 \mathrm{ng} /$ $\mathrm{mL}$ and $136.95 \mathrm{mg} / \mathrm{L}$ respectively. On descriptive statistics, P-SEP proved to be the most sensitive marker (93.3\%) for advanced COVID infection and showed the highest rate of identifying patients with underlying severe disease (76.7\%), compared to CRP and PCT. The highest specificity rate was accounted for PCT (30\%) in the current study.

On ROC analysis, a strong statistical association $(p<0.001)$ of P-SEP with severe COVID infection was seen, 
Table 1. Distribution of all participants with respect to clinicopathological characteristics and statistical estimates

\begin{tabular}{|c|c|c|c|c|c|c|c|c|}
\hline Clinical characteristics & \multicolumn{8}{|c|}{ Distribution $(n=80)$} \\
\hline Sex (male/female) & \multicolumn{8}{|l|}{$54 / 26$} \\
\hline Median age (years) & \multicolumn{8}{|c|}{$67.5(33-90)$} \\
\hline \multirow{2}{*}{$\begin{array}{l}\text { Investigations } \\
\text { (reference level) }{ }^{\dagger}\end{array}$} & \multicolumn{2}{|c|}{ The severity of COVID-19 } & \multirow{2}{*}{$\begin{array}{l}p \\
(p=0.5)^{1}\end{array}$} & \multirow{2}{*}{$\begin{array}{l}\text { Sensitivity } \\
(\%)\end{array}$} & \multirow{2}{*}{$\begin{array}{l}\text { Specificity } \\
(\%)\end{array}$} & \multirow{2}{*}{$\begin{array}{l}\text { PPV§ } \\
(\%)\end{array}$} & \multirow{2}{*}{$\begin{array}{l}\text { NPV } \neq \\
(\%)\end{array}$} & \multirow[b]{2}{*}{ AUC : } \\
\hline & $\begin{array}{l}\text { Moderate } \pi \\
(n=20)\end{array}$ & $\begin{array}{l}\text { Severe-to-critical " } \\
(n=60)\end{array}$ & & & & & & \\
\hline CRP (6 mg/L) & 19 & 54 & $0.047^{*}$ & 90 & 5 & 74 & 14.3 & 0.649 \\
\hline PCT $(0.15 \mathrm{ng} / \mathrm{mL})$ & 14 & 47 & 0.549 & 78.3 & 30 & 77 & 31.6 & 0.455 \\
\hline P-SEP (5.5 ng/L) & 17 & 56 & $0.001^{*}$ & 93.3 & 15 & 76.7 & 42.9 & 0.778 \\
\hline
\end{tabular}

reflected by the area under the curve (AUC) of 0.778 . Also, CRP levels were significantly higher $(p<0.05)$ among critically ill patients accompanied by the ROC curve (AUC) of 0.649 (Figure 1). No significant difference was recorded for PCT. When $15.99 \mathrm{ng} / \mathrm{L}$ was used as a cut-off for P-SEP, we observed $93.3 \%$ sensitivity and $85 \%$ specificity for severe infection whereas when cut-off levels for PCT; $0.156 \mathrm{ng} / \mathrm{mL}$ and CRP; $7.95 \mathrm{mg} / \mathrm{L}$ was considered, $78 \%$ sensitivity and $70 \%$ specificity for PCT while $88 \%$ sensitivity and $95 \%$ specificity for CRP was recorded.

\section{Discussion}

Recent CDC guidelines describe that the core principle behind the management of Severe Acute Respiratory syndrome-Coronavirus-2 (SARS-CoV-2) infection (COVID-19) relies on monitoring respiratory rate, oxygen saturation \& indicators of oxygenation such as; $\mathrm{PaO}_{2} / \mathrm{FiO}_{2}$. Unfortunately, the prescribed parameters are unreliable \& subjective resulting in misdiagnosis, false interpretations \& late diagnosis especially in cases with high-risk COVID-19 infection (10). So, there is an urgent need for novel bioassays that could identify the complex case or predict the clinical course of disease in order to improve outcomes among patients' by implementing appropriate treatment plans.

Measuring proinflammatory markers is considered as a cornerstone in risk assessment of patients with a wide variety of infectious diseases, due to their potential for predicting the clinical progression as well as guiding therapeutic decisions (8). Primarily, cytokine storm is the major factor behind the worst outcome among COVID-19 patients'. It is hypothesized that the interaction of macrophage and activated viral products triggers this release of cytokine and some immune mediators such as P-SEP (8). Therefore, measuring the concentration of P-SEP may provide valuable clinical information for
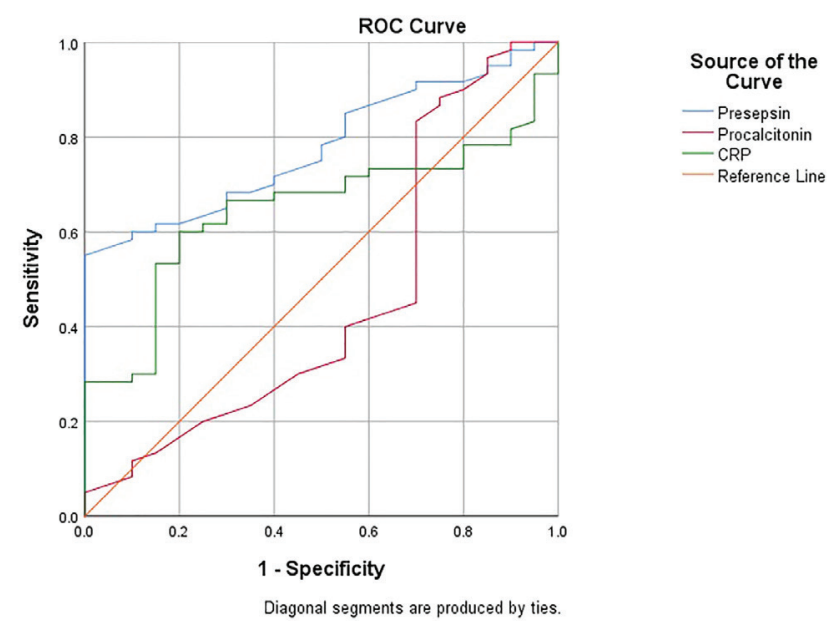

Figure 1. Receiver operating characteristic curve comparing levels of P-SEP (blue) and procalcitonin (red) and C-reactive protein (green) in severe to critical patients of COVID-19 COVID-19: Coronavirus disease-2019

risk stratification of COVID-19 patients'. In this context, recently, Domi et al. (11) and Ducastel et al. (12) showed the benefits of monitoring P-SEP in risk stratification of COVID-19 patients'. These findings are further strengthened by the results of a recent pooled analysis (8). Previously, Zaninotto et al. (3) and Fukada et al. (10) found a positive relationship of inflammatory biomarkers with COVID-19 and its clinical course in a limited set of cases and recommended further research. We, therefore, took a lead to investigate this association in a large group of patients with proven COVID-19 infection. To the best of our knowledge, this is the first original research conducted with the intent to determine the independent prognostic value of P-SEP, PCT and CRP for COVID-19 infection. Our findings may have long-lasting clinical implications. P-SEP is a recently identified biomarker which may facilitate in the 
diagnosis of sepsis. Structurally, it is a truncated $\mathrm{N}$-terminal soluble CD14 subtype (10). Whereas, PCT is a derivative of calcitonin, which is released primarily from $C$ cells of the thyroid gland, monocytes as well as hepatocytes. PCT is a well-established molecule for identifying inflammatory response of infectious origin (13). Compared to P-SEP and $P C T, C R P$ is produced by the liver in response to an ongoing inflammation anywhere in the body (14).

Numerous studies have reported the utility of P-SEP in predicting the severity and mortality in some inflammatory conditions related to infections (15). In the present research, we retrospectively compared the plasma levels of inflammatory markers including P-SEP between moderate and severe to critically ill COVID-19 patients'. The diagnosis of COVID-19 infection was performed by isolating SARSCoV-2 RNA from nasopharyngeal swab specimens while patients' clinical status was determined in accordance with CDC guidelines (2). We have determined that P-SEP were significantly higher among severe to critically ill patients on admission than those who had a moderate disease. Furthermore, P-SEP has shown better sensitivity and specificity, which suggested that this molecule could serve as a useful prognostic indicator of COVID infection. Similar findings were reported in case series by Fukada et al. (10).

Despite no established mechanism of P-SEP elevation in COVID-induced pneumonia is documented to date, several studies have shown a strong relationship of P-SEP with short-term mortality in patients of acute respiratory distress syndrome (ARDS) (16). Because the potential of P-SEP to predict the progression of ARDS is well recognized, we believe this might assist clinicians to distinguish highrisk COVID-19 patients on admission and implement appropriate treatment strategies at an early stage.

In agreement with previous studies (17-19), we came across a weak statistical association of CRP accompanied by reasonably good sensitivity. We suggest that the observed relationship of CRP could be contributed by the inflammatory environment due to its natural proinflammatory characteristic, rather than the infection itself. However, this inference is subject to confirmation.

In a recent study by Tuncer et al. (20) PCT has shown the highest odds for predicting deterioration among COVID-19 patients', however, P-SEP status was not investigated either compared with other inflammatory markers. In contrast, some previous studies had shown limited or no role of PCT in the diagnosis and prognosis of COVID-19 $(3,10)$. In the current research, although, PCT levels were elevated in the majority of cases, no difference between moderate and severe COVID-19 groups was seen. Moreover, the lowest sensitivity and specificity values were recorded for PCT in the present study. This observation is in agreement with Zaninotto et al. (3) and Fakuda et al. (10). We believe that steady and slow increase of PCT is the inherent property under infectious conditions and thus the values taken on day 0 of admission may have biased our findings. Therefore, further research is recommended in this regard.

Another important aspect of the present study is the proposed cut-off levels of inflammatory markers for risk stratification of COVID-19 infection. Previously, Zaninotto et al. (3) suggested a cut-off level of P-SEP for severe infection; however, the cut-off range of other inflammatory biomarkers was not evaluated and compared. Also, the study was unable to measure the sensitivity and specificity for the proposed P-SEP cut-off (3). Contrary to that, we suggest independent concentrations of P-SEP, PCT and CRP can be used to identify COVID cases with poor outcomes. Besides that, we also described sensitivity and specificity for corresponding cut-off concentration which gives further strength to our findings. However, we recommend more research using a larger sample size to establish precise cut-off limits for each of those molecules.

\section{Study Limitations}

Our study has some limitations. Firstly, due to consecutive sampling, the distribution of cases across two groups was not equal which might have influenced our findings. Secondly, we were not able to include cases with outcomes of death and hence were unable to assess the utility of these molecules to predict mortality. This limitation is contributed by the hospital policy of refraining the data of those who died from COVID-19. Thirdly, the study was conducted with the aim to determine the prognostic utility of inflammatory markers in COVID infection and hence only values corresponding to the day of admission were taken into account, thus preventing us to compare plasma levels with disease progression. Lastly, further studies are needed to better explain the mechanisms responsible for elevated levels of inflammatory markers especially; P-SEP in SARS-CoV-2 patients' and in particular its relation with multiorgan failure syndrome. Despite these limitations, there are certain strengths of the present research which are worth mentioning. This study is among a few studies to analyze the role of pro-inflammatory markers for the severity of COVID-19. We have conducted our research on a larger sample size compared to previous studies and performed a comprehensive comparative analysis of conventional markers and novel P-SEP. Overall, our findings may offer a useful strategy to stratify high-risk COVID-19 patients' in ICU admission who would benefit from intensive treatment.

\section{Conclusion}

The data obtained seems to demonstrate the role of the inflammatory biomarkers in providing prognostic 
information in high-risk COVID-19 patients', as already described in several other diseases. Nevertheless, our findings showed that among all analyzed molecules; P-SEP proved to be the most useful tool in predicting the severity of COVID-19 infection. Further multicenter studies with a large number of subjects are warranted to confirm our findings.

\section{Authorship Contributions}

Concept: S.S., F.A.B., Design: S.S., F.A.B., Data Collection or Processing: F.H.M., F.A.B., Analysis or Interpretation: F.A.B., Literature Search: F.A.B., Writing: F.A.B.

Conflict of Interest: No conflict of interest was declared by the authors.

Financial Disclosure: The authors declared that this study received no financial support.

\section{References}

1. Organization WH. COVID-19 weekly epidemiological update, edition 45, 22 June 2021. 2021.

2. Centers for Disease Control and Prevention. Interim clinical guidance for management of patients with confirmed coronavirus disease (COVID-19), 2020.

3. Zaninotto M, Mion MM, Cosma C, Rinaldi D, Plebani M. Presepsin in risk stratification of SARS-CoV-2 patients. Clin Chim Acta 2020;507:161-3.

4. Bohn MK, Lippi G, Horvath A, et al. Molecular, serological, and biochemical diagnosis and monitoring of COVID-19: IFCC taskforce evaluation of the latest evidence. Clin Chem Lab Med 2020;58:1037-52. doi: 10.1515/cclm-2020-0722. PMID: 32459192.

5. Afzal AJJoar. Molecular diagnostic technologies for COVID-19: Limitations and challenges. 2020.

6. Tang YW, Schmitz JE, Persing DH, Stratton CW. Laboratory Diagnosis of COVID-19: Current Issues and Challenges. J Clin Microbiol 2020;58:e00512-20

7. Bennouar S, Bachir Cherif A, Kessira A, et al. Usefulness of biological markers in the early prediction of corona virus disease-2019 severity. Scand J Clin Lab Invest 2020;80:611-8.

8. Lippi G, Sanchis-Gomar F, Henry BM. Presepsin value predicts the risk of developing severe/critical COVID-19 illness: results of a pooled analysis. Clin Chem Lab Med 2021.
9. Iwen PC, Stiles KL, Pentella MA. Safety Considerations in the Laboratory Testing of Specimens Suspected or Known to Contain the Severe Acute Respiratory Syndrome Coronavirus 2 (SARS-CoV-2). Am J Clin Pathol 2020:15;153:567-70.

10. Fukada A, Kitagawa $Y$, Matsuoka M, et al. Presepsin as a predictive biomarker of severity in COVID-19: A case series. J Med Virol 2021;93:99-101.

11. Domi H, Matsuura H, Kuroda M, Yoshida M, Yamamura $\mathrm{H}$. Simple prognostic factors and change of inflammatory markers in patients with severe coronavirus disease 2019: a single-center observational study. Acute Med Surg 2021:12;8:683.

12. Ducastel M, Chenevier-Gobeaux C, Ballaa Y, et al. Oxidative Stress and Inflammatory Biomarkers for the Prediction of Severity and ICU Admission in Unselected Patients Hospitalized with COVID-19. Int J Mol Sci 2021:12;22:7462.

13. Hayashida K, Kondo Y, Hara Y, Aihara M, Yamakawa K. Headto-head comparison of procalcitonin and presepsin for the diagnosis of sepsis in critically ill adult patients: a protocol for a systematic review and meta-analysis. BMJ Open 2017:6;7:014305.

14. Sankar V, Webster NRJJoa Clinical application of sepsis biomarkers. J Anesth 2013:;27:269-83.

15. Gasteiger S, Primavesi F, Werkl P, et al. The prognostic value of Presepsin for postoperative complications following pancreatic resection: A prospective study. PLoS One 2020:9;15:e0243510.

16. Liu B, Chen YX, Yin Q, Zhao YZ, Li CS. Diagnostic value and prognostic evaluation of Presepsin for sepsis in an emergency department. Crit Care 2013:20;17:244.

17. Chen W, Zheng KI, Liu S, Yan Z, Xu C, Qiao Z. Plasma CRP level is positively associated with the severity of COVID-19. Ann Clin Microbiol Antimicrob 2020:15;19:18.

18. Ali N. Elevated level of C-reactive protein may be an early marker to predict risk for severity of COVID-19. J Med Virol 2020;92:2409-11.

19. Wang L. C-reactive protein levels in the early stage of COVID-19. Med Mal Infect 2020;50:332-4.

20. Tuncer G, Surme S, Bayramlar OF, et al. National Early Warning Score 2 and laboratory predictors correlate with clinical deterioration in hospitalized patients with COVID-19. Biomark Med 2021;15:807-20. 